\title{
BMJ Open Preventing postnatal maternal mental health problems using a psychoeducational intervention: the cost-effectiveness of What Were We Thinking
}

Jemimah Ride, ${ }^{1}$ Paula Lorgelly, ${ }^{1,2}$ Thach Tran, ${ }^{3}$ Karen Wynter, ${ }^{3}$ Heather Rowe ${ }^{3}$ Jane Fisher ${ }^{3}$

To cite: Ride J, Lorgelly P, Tran T, et al. Preventing postnatal maternal mental health problems using a psychoeducational intervention: the costeffectiveness of What Were We Thinking. BMJ Open 2016;6:e012086.

doi:10.1136/bmjopen-2016012086

- Prepublication history and additional material is available. To view please visit the journal (http://dx.doi.org/ 10.1136/bmjopen-2016012086).

Received 29 March 2016 Revised 10 October 2016 Accepted 21 October 2016

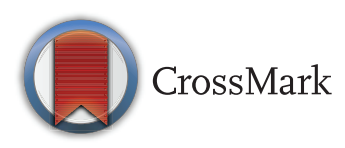

${ }^{1}$ Centre for Health Economics, Monash University, Clayton, Victoria, Australia

${ }^{2}$ Office of Health Economics, London, UK

${ }^{3}$ Jean Hailes Research Unit, Monash University,

Melbourne, Victoria, Australia

Correspondence to Dr Jemimah Ride; jemimah.ride@monash.edu

\section{ABSTRACT}

Objectives: Postnatal maternal mental health problems, including depression and anxiety, entail a significant burden globally, and finding cost-effective preventive solutions is a public policy priority. This paper presents a cost-effectiveness analysis of the intervention, What Were We Thinking (WWWT), for the prevention of postnatal maternal mental health problems.

Design: The economic evaluation, including costeffectiveness and cost-utility analyses, was conducted alongside a cluster-randomised trial.

Setting: 48 Maternal and Child Health Centres in Victoria, Australia.

Participants: Participants were English-speaking firsttime mothers attending participating Maternal and Child Health Centres. Full data were collected for 175 participants in the control arm and 184 in the intervention arm.

Intervention: $W W W T$ is a psychoeducational intervention targeted at the partner relationship, management of infant behaviour and parental fatigue.

Outcome measures: The evaluation considered public sector plus participant out-of-pocket costs, while outcomes were expressed in the 30-day prevalence of depression, anxiety and adjustment disorders, and quality-adjusted life years (QALYs). Incremental costs and outcomes were estimated using regression analyses to account for relevant sociodemographic, prognostic and clinical characteristics.

Results: The intervention was estimated to cost \$A118.16 per participant. The analysis showed no statistically significant difference between the intervention and control groups in costs or outcomes. The incremental cost-effectiveness ratios were \$A36 451 per QALY gained and \$A152 per percentagepoint reduction in 30-day prevalence of depression, anxiety and adjustment disorders. The estimate lies under the unofficial cost-effectiveness threshold of \$A55 000 per QALY; however, there was considerable uncertainty surrounding the results, with a $55 \%$ probability that WWWT would be considered costeffective at that threshold.

\section{Strengths and limitations of this study}

- The prospectively designed economic evaluation was conducted alongside a cluster-randomised controlled trial of the intervention's clinical effectiveness.

- The sample size, based on the clinical effect rather than cost-effectiveness analysis, and the cluster-randomised nature of the trial may have limited the ability to detect statistically significant differences between the control and intervention groups.

- Data collected in the trial included use of health social and early childhood services and participant out-of-pocket costs, allowing the results to be examined from a range of perspectives.

- Results presented include probabilistic sensitivity analysis and scenario analyses to allow the reader to assess the impact of key uncertainties.

Conclusions: The results suggest that, although WWWT shows promise as a preventive intervention for postnatal maternal mental health problems, further research is required to reduce the uncertainty over its cost-effectiveness as there were no statistically significant differences in costs or outcomes.

Trial registration number: ACTRN12613000506796; results.

\section{BACKGROUND}

Prevention of postnatal mental health problems is a challenge for healthcare systems internationally. Depression, anxiety and adjustment disorders are common among women who have recently given birth ${ }^{1}$ which is a population subgroup of substantial size. These disorders have a significant impact on the well-being of women at a pivotal time in the life of the mother, baby 
and wider family. ${ }^{3}{ }^{4}$ There is an associated burden on the public sector; for example, women with postnatal depression (PND) have higher use of health and other services than their non-depressed counterparts, not limited to greater use of mental health services. ${ }^{5-7}$

Research on the cost-effectiveness of interventions to prevent the development of postnatal distress, depression and anxiety has shown mixed results. A programme of structured midwife-led care was found to improve postnatal mental health without increasing costs; ${ }^{8}$ counselling and support for women at high risk of $\mathrm{PND}^{9}$ and peer support ${ }^{10}$ were found to reduce symptoms or duration of depression but at a higher cost; and postnatal support worker home visits ${ }^{11}$ were found to increase costs with no associated improvement in mental health. Preventive approaches that address the woman's relationship with her spouse or partner have shown some promise in terms of clinical effectiveness; ${ }^{12}$ however, there has been little research into the cost-effectiveness of such interventions.

What Were We Thinking (WWWT) is a psychoeducational intervention that targets the parental partner relationship, management of infant behaviour and parental fatigue. A community-based before and after controlled study found that $W W W T$ reduced postnatal mental health problems among women with no history of psychiatric disorders. ${ }^{13}$ The Sleep, Parenting and Relationships in a Community Setting (SPARCS) trial was subsequently conducted to examine the clinical and cost-effectiveness of WWWT for the prevention of depression, anxiety and adjustment disorders in women at 6 months postpartum. ${ }^{14}$ The intervention was delivered soon after birth to first-time parents (FTPs) in the setting of Maternal and Child Health Centres (MCHCs) in Victoria, Australia. MCHCs are a publicly funded universal service for families of children up to school age, with the goal to "promote healthy outcomes for children and their families' (ref. 15, p. 6). High participation rates are a stated policy objective in pursuance of this goal; more than $90 \%$ of families with recent births in Victoria attend MCHCs. ${ }^{16}$ One element of the Maternal and Child Health (MCH) service is FTP group sessions, which focus on parent-child interactions, parenting confidence, emotional well-being and support for FTPs. ${ }^{17}$

Public sector decision-makers have made a commitment to using evidence to inform modifications to the $\mathrm{MCH}$ service model. ${ }^{18} \mathrm{~A}$ complexity of developing appropriate evidence is that in Victoria, early childhood service costs (including MCHCs) fall under the Department of Education and Training (formerly the Department of Education and Early Childhood Development). This means that funding of MCHCs is shared between the Department of Education and Training and Local Governments, rather than sitting within health, despite the clear health focus of the MCH service.

Economic evaluation provides decision-makers with evidence on how best to allocate limited resources by comparing the alternatives in terms of costs and outcomes. In cost-effectiveness analyses (CEA), outcomes are expressed in natural or clinical units, such as symptom improvement or life years saved. In cost-utility analyses (CUA), outcomes are expressed in terms of quality-adjusted life years (QALYs), which take into account differences in life span and health-related quality of life (HRQoL). QALYs are generally preferred in health economic evaluations as they facilitate allocative decisions between interventions with different outcomes, ${ }^{19}{ }^{20}$ and there are data to guide what might be considered cost-effective in terms of QALYs. However, studies in prevention of postnatal mental health problems often use condition-specific clinical end points, such as cost per month of PND averted. ${ }^{9}$ It is not clear what societies would be willing to pay for prevention in terms of these clinical end points. One US study found individuals were willing to pay $\sim 9 \%$ of their monthly household income for treatment of depression, ${ }^{21}$ and a German study found that people were willing to pay personally for prevention of depression; ${ }^{22}$ however, neither informs us of societal-level willingness-to-pay (WTP) for prevention of depression. Although not an official threshold, US\$50 000 per QALY (approximately $\$ A 55000$ in 2014) is often used as a guide, which could vary with factors such as budget constraints or special consideration of certain populations or diseases. ${ }^{23}$ Other thresholds have been proposed, and vary considerably; ${ }^{24}$ one study found that Australian WTP for one QALY gain was approximately $\$ A 75000,{ }^{25}$ while in the UK, a threshold of £20 000-30 000 (approximately \$A41 000-61 000) per QALY gained is used, ${ }^{26}$ although Claxton et $a l^{27}$ suggest it should be lower, approximately \$A31000 (figures adjusted to 2014 terms).

This paper describes the results of an economic evaluation of WWWT conducted alongside the SPARCS trial. The protocols for the clinical study ${ }^{14}$ and economic evaluation ${ }^{28}$ have been published previously, as have the trial results. ${ }^{29}$ This paper presents the results of a CEA and a CUA, and discusses the implications of these results for decision-makers seeking cost-effective interventions for the prevention of postnatal mental health problems.

\section{METHODS}

The economic evaluation took a public sector perspective (incorporating healthcare, early childhood and social service costs), plus the participant's out-of-pocket (OOP) costs. The time horizon of 6 months mirrored the trial follow-up period. No discounting was necessary due to the duration of the follow-up period. Ethical approval was provided by the Southern Health (now Monash Health) Human Research Ethics Committee (11388B) and the Education and Policy Research Committee, Victorian Government Department of Education and Early Childhood Development (now Department of Education and Training) (2012 001472), and the study was registered with the Monash University 
Human Research Ethics Committee (CF12) 1022-20122000474). Data were collected from participants via computer-assisted telephone interview at baseline (6 weeks postpartum) and follow-up (26 weeks postpartum).

\section{Sample and setting}

The trial was conducted in Victoria, Australia, with MCHCs as the unit of cluster randomisation. To check for similarity between participants within clusters, which could adversely affect the trial's ability to detect a difference between trial arms, the intracluster correlation coefficient (ICC) $)^{30}$ is reported for cost and outcome variables. Participants were English-speaking first-time mothers who had recently given birth and attended participating MCHCs. Baseline participant characteristics were compared between trial arms using two-sample t-tests and Pearson's $\chi^{2}$ tests.

\section{Intervention}

The training programme for nurses at intervention MCHCs comprised online and face-to-face components, with nurses who worked at more than one centre excluded to avoid cross-contamination between the intervention and control groups. Participants at intervention centres received WWWT-informed care during $\mathrm{MCH}$ visits, and both parents were invited to attend an extra 6-hour FTP group session on a Saturday, during which the $W W W T$ intervention was delivered, and received printed materials to take home. Participants at control MCHCs were provided usual MCH care, and were offered no extra FTP group sessions.

\section{Resource use and cost}

Participants were sent a card at baseline on which to record the use of health and other services during the follow-up period for themselves or their baby (specifying the service type and cost including OOP cost), and these data were collected at follow-up. This card and the service use questions asked at follow-up were developed specifically for the trial. Resource unit costs were identified from the Medicare Benefits Schedule (MBS), ${ }^{31}$ National Efficient Price (NEP) determination weights for hospital episodes, ${ }^{32}$ the standard hourly rate of MCH service provision, ${ }^{18}$ participants' OOP costs and market rates (for further detail, see online supplementary material). All unit costs were in 2013-2014 Australian dollars (\$A).

The estimated cost of the intervention per participant was based on the costs of developing the intervention, training $\mathrm{MCH}$ nurses in the intervention and delivery of the $W W W T$ session as an additional FTP group session. It was assumed that WWWT-informed MCHC care would entail the same cost per visit as standard care, with any difference in the number of visits or phone calls captured in participants' service use history. The cost of delivering the intervention to participants was based on the hourly rate of $\mathrm{MCH}$ services. In the base case, it was assumed that $\mathrm{MCH}$ nurses would need retraining after 3 years, and that six families would attend each FTP group session on average.

\section{Outcomes}

For CEA, the outcome was 30-day prevalence of depression, anxiety and adjustment disorders according to the criteria of the Diagnostic and Statistical Manual of Mental Disorders, Fourth Edition (DSM-IV) ${ }^{33}$ For CUA, the outcome was QALYs, with HRQoL measured using the three-level version of the EQ-5D (EQ-5D-3L), and calculated using Australian weights derived using the time trade off approach. ${ }^{34}$ QALYs were calculated as a linear change in HRQoL over time between baseline and follow-up.

The EQ-5D is a multiattribute utility instrument ${ }^{35}$ that assesses HRQoL over five domains of health: mobility, self-care, usual activities, pain/discomfort and anxiety/ depression. A score of one reflects full health, and zero, death. It is the most frequently used HRQoL measure when estimating QALYs, ${ }^{36}$ and has been used in the postnatal context. ${ }^{37-40}$ Petrou $e t a l^{11}$ found, among women at 6 months postpartum, an average EQ-5D score of 0.861 (95\% CI 0.844 to 0.877$)$, and a statistically significant correlation with PND risk.

\section{Analysis}

Costs and outcomes were adjusted using regression modelling to account for relevant sociodemographic, prognostic and clinical characteristics, including those differing between groups, with the effect of the intervention captured by the inclusion of the trial arm as a variable. All regression models included baseline measures of the participant's psychological well-being and psychiatric history and a measure of the baby's crying behaviour, to account for differences in baseline risks for postnatal mental health problems, plus the baby's age at baseline to account for timing of data points. In addition, the cost model included factors that might influence utilisation of health and other services: level of education, health care card status (a health care card provides access to concessions on pharmaceuticals and medical services for low-income earners and those receiving government benefits) and relative socioeconomic disadvantage of the participant's area of residence. $^{42}$ The outcome models included baseline EQ-5D-3L score to account for baseline HRQoL, plus other factors which might influence quality of life: perceived quality of the intimate partner relationship and level of support, and a measure of adverse life events (for further details regarding measures used in the study, see ref. 29). Analyses were performed in STATA V.13 using a generalised linear regression model (GLM) for cost, to allow for the non-normal distribution of costs without requiring transformation of the cost variable,$^{43} \mathrm{a}$ linear regression model for QALYs and a logit model for the binary clinical outcome (presence or absence of depression, anxiety and adjustment disorders). The 
specifications of the GLM model for costs were informed by a modified Parks test, resulting in a log link and gamma family, ${ }^{43}$ while the distribution of QALYs fitted with the assumption of normality in the linear regression model. Since these regression models included different explanatory variables than those included in the clinical evaluation, ${ }^{29}$ the results may differ slightly. Cluster-robust SEs were used to account for randomisation at the level of the MCHC, rather than the individual.

The main analysis was conducted on participants for whom complete data were collected, and repeated with imputation of missing data. Characteristics of those with missing data were compared with the complete cases using two-sample t-tests and Pearson's $\chi^{2}$ tests. Missing values for cost, outcomes and two characteristics (adverse life events and levels of support) used in regression analyses were imputed using the 'mi impute chained' command in STATA with V.30 imputations. Predictive mean matching was used for continuous variables, with logistic regression for binary and ordered logistic regression for ordinal variables, using an imputation model that included characteristics associated with missingness of data as well as the variables in the analytic model.

The incremental cost-effectiveness ratio (ICER) compares the control and intervention groups by dividing the difference in cost by the difference in outcome (each calculated using average marginal effects from the relevant regression model). Uncertainty surrounding the ICER was estimated using non-parametric bootstrapping $^{44}$ (resampling with replacement from the original data to produce replicates of the sample), resampling clusters rather than individuals. The distribution of the resulting 2000 estimates of the ICER on the costeffectiveness plane ${ }^{45}$ depicts the joint uncertainty surrounding costs and outcomes. Estimates in the lower right quadrant of a cost-effectiveness plane suggest that the intervention is more effective and less costly, while estimates in the upper right quadrant suggest it is more effective but costlier. Estimates in this quadrant require further analysis to determine if the decision-maker would be willing to allocate the extra expenditure for the extra benefit. The cost-effectiveness acceptability curve $^{46}$ is used for that purpose, to display the probability that the intervention is cost-effective at a given societal WTP threshold (ie, the proportion of the bootstrapped estimates that fall below the threshold) over a range of thresholds.

\section{Scenario analyses}

Cost per QALY was re-estimated under alternative scenarios to explore the results' sensitivity to particular assumptions or parameters. (1) Using only variables collected at baseline to adjust cost and outcomes; that is, excluding support and adverse life event variables from the regression models, which were collected at follow-up. (2) Assuming that MCH nurses would require retraining in the intervention after 1 year (instead of 3 years as in the base case). (3) Assuming that the average number of families per session would be 5 or 7 (rather than 6 as in the base case). (4) Limiting the cost analysis to the health sector, the early childhood sector or combined public sector without participant OOP costs. (5) To test the sensitivity of the results to variation in costs and outcomes, for a worst-case scenario, incremental costs were assumed to be at the upper end of the $95 \%$ CI and incremental outcomes at the lower end for each individual, while for a best-case scenario, the reverse assumptions were applied. (6) To test the sensitivity of the results to the specifications of the models, linear regression was used for all three models.

\section{EQ-5D-3L scores across clinical states}

We also explored the usefulness of the EQ-5D-3L for discriminating between clinical states in postnatal mental health, using the Mann-Whitney $\mathrm{U}$ test to investigate whether EQ-5D-3L scores differed significantly between participants with and without major depressive disorder, and those with and without symptoms of anxiety or depression. The Mann-Whitney U test ${ }^{47}$ was used because the EQ-5D-3L scores were (as expected) non-normally distributed, with a skew to the upper limit of 1 , and so would not meet the assumptions for a two-sample t-test.

\section{RESULTS}

\section{Baseline}

Cluster randomisation resulted in the allocation of 196 participants across 24 MCHCs to the control arm and 204 participants across 24 MCHCs to the intervention arm (although one MCHC in the control arm did not recruit any participants). Overall, 21 participants $(10.7 \%)$ from the control arm and $20(9.8 \%)$ from the intervention arm did not complete the full follow-up interview, leaving 175 in the control arm and 184 in the intervention arm for complete case analysis. Baseline interviews took place between May 2013 and April 2014, and follow-up interviews between September 2013 and August 2014.

Participants' baseline characteristics, outlined in table 1, were similar between groups except that the intervention group had higher depression $(\mathrm{p}=0.045)$ and anxiety $(\mathrm{p}=0.004)$ scores and more participants with a history of eating or dieting disorders $(p=0.039)$, while the control group reported a higher number of hours that the baby cried or fussed per day $(p=0.048)$. These variables were included in the regression models for costs and outcomes, along with other relevant characteristics.

The pattern of missing data was monotone, since close to $100 \%$ of data were non-missing at the initial interview, and most data missing at follow-up involved the entire interview. Data were not missing completely at random, as those with missing data were more likely to be born overseas, speak a language other than English, hold a health care card had lower levels of education and gave lower self-reported health ratings, but were no different in psychiatric history. These characteristics were included 
Table 1 Baseline participant characteristics

\begin{tabular}{|c|c|c|c|}
\hline $\begin{array}{l}\text { Characteristic at baseline } \\
\mathrm{N}\end{array}$ & $\begin{array}{l}\text { Control group } \\
175 \\
\text { Mean (SD) }\end{array}$ & $\begin{array}{l}\text { Intervention group } \\
184 \\
\text { Mean (SD) }\end{array}$ & $\begin{array}{l}\text { p Value test of difference } \\
\text { t-test }\end{array}$ \\
\hline Mean age, years & $31.3(5.12)$ & $31.0(5.10)$ & 0.563 \\
\hline EQ-5D score & $0.859(0.14)$ & $0.858(0.15)$ & 0.910 \\
\hline Socioeconomic index for areas ${ }^{1}$ & $1009.57(30.12)$ & $1014.43(40.08)$ & 0.197 \\
\hline Depression score ${ }^{2}$ & $3.46(3.27)$ & $4.20(3.61)$ & 0.045 \\
\hline Anxiety score ${ }^{2}$ & 2.77 (2.97) & $3.85(3.90)$ & 0.004 \\
\hline \multirow[t]{2}{*}{ Number of hours baby fusses or cries per 24 hours } & $3.30(2.52)$ & $2.83(1.92)$ & 0.048 \\
\hline & N (\%) & N (\%) & Pearson $\chi^{2}$ test \\
\hline Country of birth & & & 0.794 \\
\hline Australia & $138(78.86)$ & $143(77.72)$ & \\
\hline Language spoken at home & & & 0.310 \\
\hline English & $145(82.86)$ & $159(86.41)$ & \\
\hline English+other & $21(12.00)$ & $21(11.41)$ & \\
\hline Other & $9(5.14)$ & $4(2.17)$ & \\
\hline Level of education & & & 0.738 \\
\hline University degree & $115(65.71)$ & $114(61.96)$ & \\
\hline TAFE or college certificate or diploma & $33(18.86)$ & $37(20.11)$ & \\
\hline Up to completion of high school & $27(15.43)$ & 33 (17.93) & \\
\hline Marital status & & & 0.083 \\
\hline Married or de facto & 165 (94.29) & $180(97.83)$ & \\
\hline Single or not living with partner & $10(5.71)$ & $4(2.17)$ & \\
\hline Health care card holder & $26(14.86)$ & $18(9.78)$ & 0.143 \\
\hline Private health insurance & $124(70.86)$ & $113(61.41)$ & 0.059 \\
\hline Self-rated health & & & 0.706 \\
\hline Excellent & $62(35.43)$ & $64(34.78)$ & \\
\hline Very good & $89(50.86)$ & 89 (48.37) & \\
\hline Good & $24(13.71)$ & $31(16.85)$ & \\
\hline \multicolumn{4}{|l|}{ History of... } \\
\hline Any psychiatric disorder & $37(21.14)$ & $52(28.26)$ & 0.118 \\
\hline Depression & $24(13.71)$ & $37(20.11)$ & 0.107 \\
\hline Anxiety & $25(14.29)$ & $30(16.30)$ & 0.596 \\
\hline Post-traumatic stress disorder & $6(3.43)$ & $4(2.17)$ & 0.470 \\
\hline Eating or dieting disorder & $2(1.14)$ & $9(4.89)$ & 0.039 \\
\hline Alcohol or drug dependence & $1(0.57)$ & $4(2.17)$ & 0.195 \\
\hline Physical or sexual abuse & $10(5.71)$ & $18(9.78)$ & 0.151 \\
\hline
\end{tabular}

in the model for multiple imputation of missing data in scenario analysis.

The mean baseline EQ-5D-3L utility score was 0.859 , with no statistically significant difference between the groups. However, in answering the anxiety/depression EQ-5D-3L domain question, more of the intervention group $(20 \%)$ reported moderate anxiety or depression than the control group $(13 \%)(\mathrm{p}=0.054)$.

\section{Outcomes}

At follow-up, the unadjusted 30-day prevalence of DSM-IV diagnoses of depression, anxiety and adjustment disorders was $8.57 \%$ in the control group, and $8.70 \%$ in the intervention group $(\mathrm{p}=0.967, \mathrm{ICC}<0.001)$.

EQ-5D-3L scores at follow-up were also similar between the control $(0.890)$ and intervention groups (0.889) $(\mathrm{p}=0.980)$, with $23 \%$ of the control group and
$20 \%$ of the intervention group reporting moderate anxiety/depression, and $2 \%$ of the intervention group reporting extreme anxiety/depression (none in the control group) $(\mathrm{p}=0.107)$.

Individual QALYs were calculated by dividing the mean of the two EQ-5D-3L scores for each person by their duration of follow-up, which varied from 78 to 288 days depending on the baby's age at recruitment (1.1-15.6 weeks) but was similar between groups $(p=0.779)$. The mean QALY accumulated for the control group was 0.371 and for the intervention group was $0.372(\mathrm{p}=0.883$, ICC 0.034$)$.

After regression modelling to account for relevant sociodemographic, prognostic and clinical characteristics, the differences between groups were still not statistically significant; using complete case analysis, the intervention group had a 1.77 percentage-point lower 
prevalence of depression, anxiety and adjustment disorders $(p=0.497)$ and 0.007 higher QALYs accrued $(\mathrm{p}=0.417)$ than the control group. From the analysis using multiple imputation of missing data, the intervention group had 0.33 percentage-point lower prevalence $(\mathrm{p}=0.643)$ and 0.006 higher QALYs $(\mathrm{p}=0.544)$.

\section{Costs}

The estimated intervention cost per participant was \$A118.16 in the base case. This included MCH nurse time to deliver the intervention (based on an average of 6 families attending each WWWT 6-hour session) and to complete the 3-hour self-directed training and the day and a half of face-to-face training in the intervention. It also included development costs to account for previous research conducted in developing $W W W T$. Average perparticipant intervention costs were calculated as if the intervention were to be delivered state-wide, rather than using the numbers in attendance during the trial, as the intervention was delivered as routine at intervention $\mathrm{MCH}$ centres. The intervention MCH centres delivered WWWT-informed care to all women at the centres, as it was randomised at the centre level, and more women attended WWWT sessions than the number who enrolled in the trial, so the approach had to vary from the usual per-participant trial cost estimation. For the trial, $33 \mathrm{MCH}$ nurses were trained in the WWWT approach, and a total of 44 seminars were delivered by these nurses to an average of 4 families per session. Almost half $(46 \%)$ of women in the intervention arm of the trial attended a seminar (further details of the implementation of the intervention in the trial are available in Fisher et $a .^{29}$ )

Table 2 outlines the service use accrued during the period of follow-up. There were no statistically significant differences in service use between the intervention and control groups.

As shown in table 3, costs of health and other services were $\$ A 158.37$ higher per participant $(p=0.580$, ICC 0.002) in the intervention than control group, mostly arising from statistically insignificant higher health service costs in the intervention group. Inclusive of intervention costs, the difference was $\$ \mathrm{~A} 276.53$, and although this difference was not statistically significant, the intervention cost component was an addition to usual care costs.

Table 2 Service use during the follow-up period

\begin{tabular}{|c|c|c|c|c|c|}
\hline \multirow[b]{2}{*}{ Service type } & \multicolumn{2}{|c|}{ Control $(\mathrm{N}=175)$} & \multicolumn{2}{|c|}{$\begin{array}{l}\text { Intervention } \\
(\mathrm{N}=184)\end{array}$} & \multirow{2}{*}{$\begin{array}{l}\text { Two-sided } \\
\text { t-test } \\
\text { p Value }\end{array}$} \\
\hline & No. & Mean & No. & Mean & \\
\hline \multicolumn{6}{|l|}{$\mathrm{MCH}$ services } \\
\hline $\mathrm{MCH}$ visit & 793 & 4.5 & 800 & 4.4 & 0.581 \\
\hline $\mathrm{MCH}$ home visit & 74 & 0.4 & 89 & 0.5 & 0.612 \\
\hline $\mathrm{MCH}$ telephone line & 192 & 1.1 & 232 & 1.3 & 0.581 \\
\hline First-time parents' group & 845 & 4.8 & 924 & 5.0 & 0.525 \\
\hline \multicolumn{6}{|l|}{ Mental health } \\
\hline Psychologist, psychiatrist or counsellor & 105 & 0.6 & 108 & 0.6 & 0.966 \\
\hline Psychiatric mother-baby unit admission & 0 & 0.0 & 2 & 0.0 & 0.167 \\
\hline Web therapy session & 16 & 0.1 & 106 & 0.6 & 0.289 \\
\hline Antidepressant, anxiolytic or sedative & 11 & 0.1 & 20 & 0.1 & 0.161 \\
\hline \multicolumn{6}{|l|}{ Primary care } \\
\hline GP & 516 & 2.9 & 623 & 3.4 & 0.213 \\
\hline Clinic nurse & 27 & 0.2 & 42 & 0.2 & 0.324 \\
\hline Nurse-on-call (telephone helpline) & 59 & 0.3 & 70 & 0.4 & 0.599 \\
\hline \multicolumn{6}{|l|}{ Medical } \\
\hline Obstetrician & 67 & 0.4 & 88 & 0.5 & 0.232 \\
\hline Paediatrician & 117 & 0.7 & 115 & 0.6 & 0.764 \\
\hline Other specialist & 12 & 0.1 & 12 & 0.1 & 0.924 \\
\hline Allied health (physiotherapist, dietician or social worker) & 197 & 1.1 & 268 & 1.5 & 0.316 \\
\hline Complementary healthcare & 266 & 1.5 & 254 & 1.4 & 0.693 \\
\hline \multicolumn{6}{|l|}{ Hospital-related } \\
\hline Outpatients & 85 & 0.5 & 105 & 0.6 & 0.597 \\
\hline Emergency department visit & 47 & 0.3 & 54 & 0.3 & 0.725 \\
\hline Total days hospital admission & 71 & 0.4 & 35 & 0.3 & 0.320 \\
\hline \multicolumn{6}{|l|}{ Early parenting services } \\
\hline Residential early parenting centre & 6 & 0.0 & 6 & 0.0 & 0.940 \\
\hline Early parenting centre day stay & 16 & 0.1 & 17 & 0.1 & 0.976 \\
\hline Breastfeeding telephone helpline & 85 & 0.5 & 90 & 0.5 & 0.979 \\
\hline $\begin{array}{l}\text { Lactation consultant, mothercraft nurse, support group or } \\
\text { other parenting service }\end{array}$ & 145 & 0.8 & 157 & 0.9 & 0.922 \\
\hline
\end{tabular}


Table 3 Unadjusted costs by sector $(\$ A, 2014)$

\begin{tabular}{llccc}
\hline Cost sector & Control & Intervention & Difference & \\
Mean (SE) & Mean (SE) & Two-sided t-test \\
\hline Public sector & Mean (SE) & $1428.60(190.19)$ & $74.93(267.79)$ & $\mathrm{p}=0.780$ \\
Health & $1353.66(188.18)$ & $847.95(156.50)$ & $71.11(235.02)$ & $\mathrm{p}=0.762$ \\
Early childhood & $776.84(176.06)$ & $438.80(15.76)$ & $9.41(26.87)$ & $\mathrm{p}=0.727$ \\
Human services & $429.40(22.02)$ & $141.85(61.41)$ & $-5.58(79.90)$ & $\mathrm{p}=0.944$ \\
Participant out-of-pocket & $147.43(50.43)$ & $377.29(42.07)$ & $33.52(52.88)$ & $\mathrm{p}=0.527$ \\
Total (non-intervention) & $343.77(31.28)$ & $1805.89(209.49)$ & $158.37(285.50)$ & $\mathrm{p}=0.580$ \\
Intervention cost & $1647.52(192.67)$ & 118.16 & 118.16 & $\mathrm{p}=0.333$ \\
Total cost & - & $1924.05(209.49)$ & $276.53(285.50)$ & \\
\hline
\end{tabular}

After adjustment using regression modelling and inclusive of costs relating to the intervention, with complete case analysis, the intervention group had \$A268.69 additional costs $(\mathrm{p}=0.335)$ per participant; with imputation of missing data, the estimated additional cost was $\$ A 249.75(\mathrm{p}=0.311)$.

\section{Cost-effectiveness}

While neither costs nor outcomes demonstrated statistically significant differences between groups, a point estimate of the ICER can still be calculated based on the trial results, and can be informative as the best available estimate of cost-effectiveness, ${ }^{48}$ albeit with a high degree of uncertainty surrounding the result. Using complete case analysis, the estimated cost per percentage-point reduction in 30-day prevalence of depression, anxiety and adjustment disorders was \$A152 (95\% CI -16453 to 16756 ). The estimated cost per QALY was $\$$ A36 451 (95\% CI -1554006 to 1626908 ). As shown in figure 1, the bootstrapped estimates of incremental costs and QALYs were in all four quadrants, reflecting the uncertainty both costs and effectiveness. The majority $(69 \%)$ fell in the northeast quadrant, suggesting that the intervention might be costlier and more effective. The bootstrapped estimates for the clinical effectiveness outcome showed a similar pattern (available as online supplementary figure $\mathrm{S} 1$ ).

At a threshold of $\$ A 55000$ per QALY, there is a $55 \%$ probability that the intervention is cost-effective. (Cost-effectiveness acceptability curve is available as online supplementary figure S2.) At a threshold of $\$ A 100000$ per QALY, the probability only rises by a small increment (to $67 \%$ ) due to the uncertainty surrounding the effectiveness of the intervention.

Using the analysis that imputed missing values (intention-to-treat analysis), the estimated cost per percentage-point reduction in 30-day prevalence of depression, anxiety and adjustment disorders was \$A747 and the estimated cost per QALY was \$A44 830. These higher ICERs were driven by smaller differences in outcomes in the imputed data set. Using this approach, at the $\$ 55000$ per QALY threshold, there

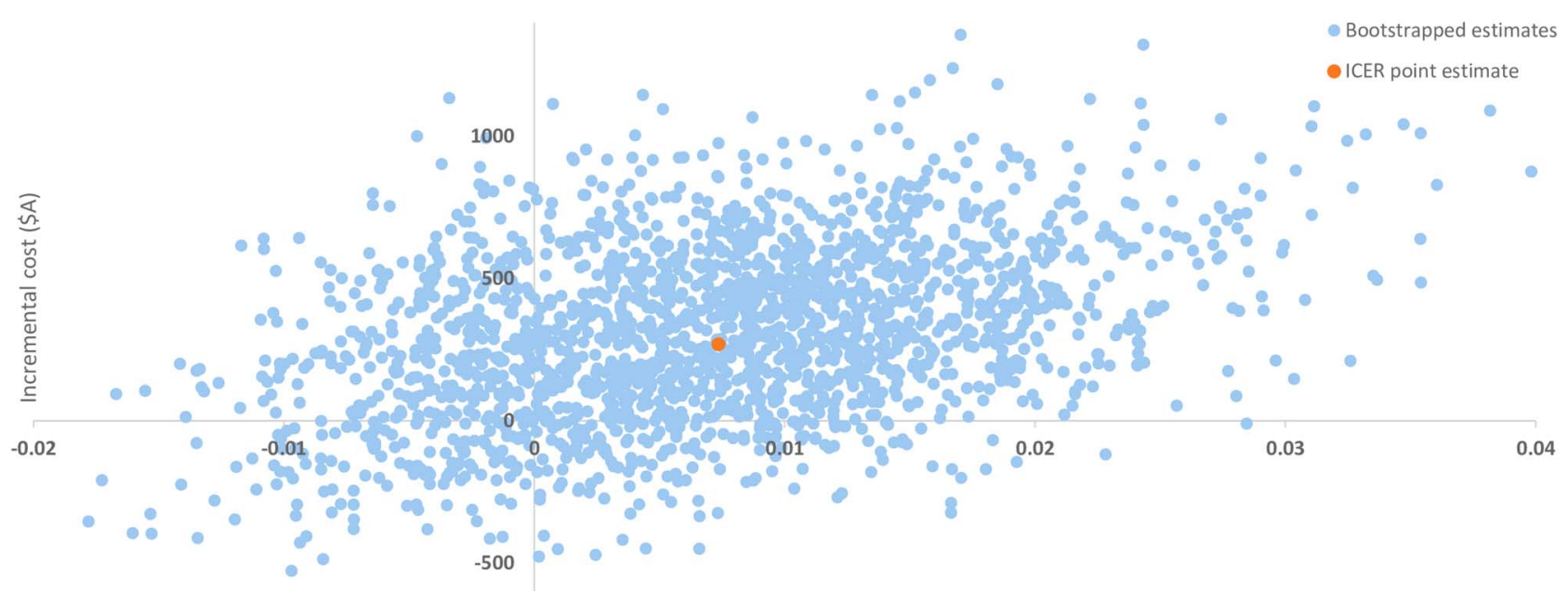

$-1000$

Incremental QALY

Figure 1 Cost-effectiveness plane-cost per quality-adjusted life year. 
Table 4 Scenario analyses: cost-effectiveness $(\$ A, 2014)$

\begin{tabular}{llll}
\hline & \multicolumn{2}{l}{ Incremental cost-effectiveness ratio (ICER) } & \\
\cline { 2 - 3 } & $\begin{array}{l}\text { Cost per } \\
\text { quality-adjusted } \\
\text { life year (CUA) }\end{array}$ & $\begin{array}{l}\text { Cost per \%-point reduction } \\
\text { in 30-day prevalence of } \\
\text { depression, anxiety and } \\
\text { adjustment disorders (CEA) }\end{array}$ & $\begin{array}{l}\text { Probability of } \\
\text { cost-effectiveness at } \\
\text { threshold of \$A55 000 }\end{array}$ \\
per QALY (\%)
\end{tabular}

was a $53 \%$ probability that the intervention is cost-effective.

\section{Scenario analyses}

The estimated ICERs under each scenario shown in table 4 demonstrate the potential impact of different methodological approaches or perspectives. Since most of the difference in non-intervention costs arose from the use of health rather than other services, limiting the perspective to the health sector gave a higher ICER than the base case. Limiting it to costs attributable to the early childhood sector (which is responsible for funding MCH services in Victoria) shows a much more favourable ICER. Participant OOP costs contributed to the cost difference, so that a public sector perspective (which excludes these) gave a lower ICER. The use of a GLM model for costs and logit model for the clinical outcome, chosen because of their superior theoretical basis, produced a higher ICER than when linear regression models were used.

\section{EQ-5D-3L scores across clinical states}

There was a gradient of EQ-5D-3L scores over increasing severity of depression and anxiety symptoms, as shown in figure 2. The mean EQ-5D-3L scores were found to differ significantly between those with $(0.682)$ and without (0.878) major depressive disorder $(\mathrm{p}<0.001)$, and those with (0.806) and without (0.917) symptoms of depression or anxiety $(\mathrm{p}<0.001)$.

\section{DISCUSSION}

This study found that $W W W T$ may be associated with higher costs and a small improvement in HRQoL and reduced prevalence of PND, anxiety and adjustment disorders when compared with usual MCH care, although
Figure 2 The mean EQ-5D-3L scores by severity of $\mathrm{PHQ}$ depression and anxiety scores. ${ }^{*} \mathrm{PHQ}$, Patient Health Questionnaire.

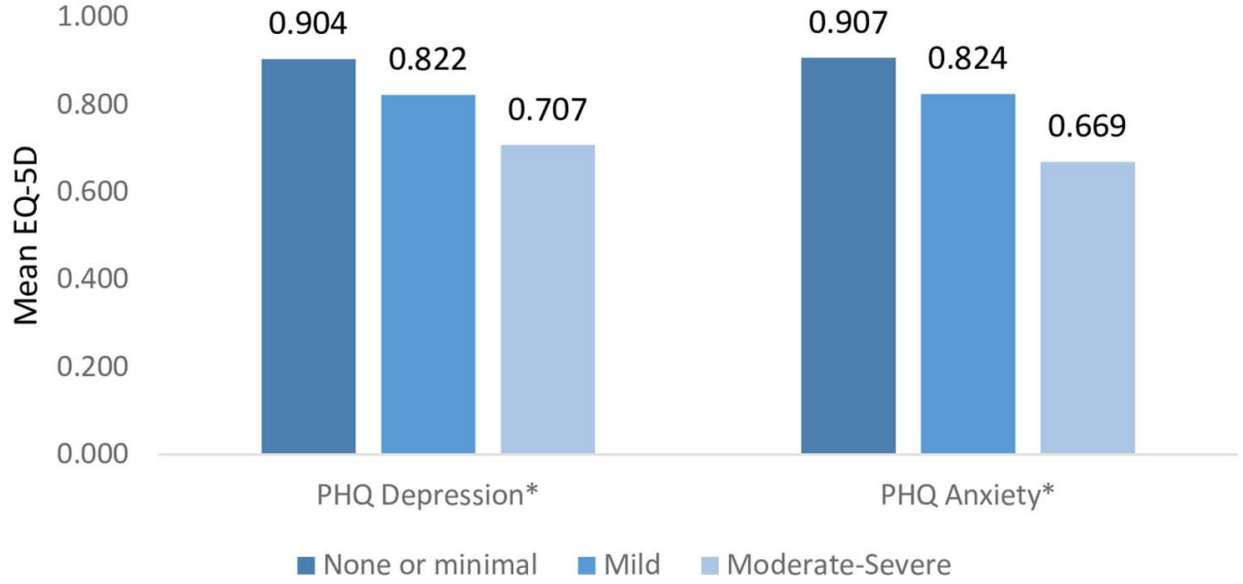


these differences were not statistically significant. We focus on the findings regarding cost per QALY gained, since there is no generally accepted level of WTP for one unit of improvement in the clinical outcome, and there is more acceptance of cost per QALY evidence by decision-makers.

The point estimate of the ICER, \$A36 451 per QALY gained, was lower than the unofficial threshold of $\$ A 55000,{ }^{23}$ as were all but one of the higher ICERs estimated under differing assumptions in the scenario analyses outlined in table 4 . There was considerable uncertainty surrounding this result, with only a $55 \%$ probability that the intervention was cost-effective at a threshold of \$A55 000 per QALY. The large differences in ICER dependent on which public sector costs were included are of particular importance since $\mathrm{MCH}$ services in Victoria are funded with early childhood services, while the intervention might be considered to fit better within a health sector perspective.

Other CEA of preventive interventions in postnatal mental health have evaluated targeted rather than universal preventive interventions and/or have used differing clinical end points as the measure of effectiveness, leading to difficulties in comparing results. For example, the PoNDER trial, ${ }^{49}$ which used QALYs as an outcome measure but targeted women at risk of PND, found no statistically significant difference in costs or QALYs and a probability of cost-effectiveness of around $80 \%$ at a threshold of $£ 20000-30000$ per QALY gained. A Canadian study, ${ }^{10}$ which investigated a programme of peer support for high-risk women in terms of cost per case of PND averted (rather than cost per QALY), found the point estimate of the ICER to be \$CAD10 009 per case averted. In a UK study ${ }^{9}$ focusing on counselling and support for women at risk of PND, no statistically significant differences were found in costs and outcomes, with a probability of cost-effectiveness of $71 \%$ at a threshold of $£ 1000$ per month of PND averted.

While this study did not demonstrate savings in unadjusted health service utilisation associated with the intervention, the intervention group had statistically significantly worse baseline depression and anxiety scores but not (as might have been expected) statistically significantly higher health service costs. This finding in relation to the use of residential early parenting centres may be of particular interest to the early childhood sector, since these are high-cost services and have similar risk factors to those addressed by the intervention. ${ }^{50}$

The data were not missing completely at random, suggesting that intention-to-treat analysis with imputation of missing values may be appropriate. However, it is not possible to tell from the observed data whether the pattern of missingness also depends on the outcome variable, and under some patterns of missingness (involving correlation with covariates), complete case analysis may be unbiased. Therefore, complete case and intention-to-treat analyses are presented. ${ }^{51}$
The correlation we found between EQ-5D-3L score and postnatal mental health supports previous findings that the EQ-5D-3L is able to discriminate between clinically significant mental health states. ${ }^{52}{ }^{53}$ We found statistically significant differences in EQ-5D-3L scores based not only on diagnosis of major depressive disorder, but also based on symptoms of depression or anxiety (a lower clinical threshold).

\section{Limitations and possibilities for further research}

Sample size calculations were based on the clinical effect rather than cost-effectiveness analysis, ${ }^{54} 55$ which may have contributed to uncertainty surrounding the results. The cluster-randomised nature of the trial and small but non-negligible ICC for QALYs may also have reduced the ability to detect an effect of the intervention on QALYs in this trial. ${ }^{30}$

No extrapolation beyond the 6-month time horizon of the trial was conducted, since there were no statistically significant differences in this period. However, significant differences between the intervention and control groups may have emerged with a longer follow-up period.

This evaluation focused on costs of public sector services and OOP costs for mothers and infants. Since postnatal mental health problems in couples tend to co-occur, ${ }^{56}$ and since the intervention explicitly included participants' partners and addressed the partner relationship, future research into WWWT could include partners' costs and outcomes, including partners' productivity. In the postnatal context, mothers' workforce participation may be low even in the absence of mental health problems, ${ }^{57}$ which may limit the relevance of productivity in terms of mothers' employment.

The cost analysis depended on participants' recall of service use. To minimise recall error, participants were asked to record services used during the follow-up period and were prompted by interviewers to refer to this record and to report use of a wide range of services.

In this study, $W W W T$ was evaluated as additional to standard FTP group sessions. However, there is a range of standard sessions available, from which MCHCs choose. If $W W W T$ were incorporated into this range, the cost would be absorbed into usual FTP group costs but other content would be displaced. Future research could estimate the cost-effectiveness of $W W W T$ in head-to-head comparison with other FTP group sessions rather than as an additional session.

Research into the cost-effectiveness of WWWT using alternative delivery mechanisms (such as online), and WTP (societal and/or decision-makers') for prevention of postnatal mental health problems could also be valuable to decision-makers.

\section{CONCLUSIONS}

This economic evaluation conducted alongside a clusterrandomised trial found no statistically significant 
difference in either costs or effectiveness, with a point estimate of cost-effectiveness that suggests $W W W T$ might be a cost-effective intervention for the prevention of postnatal mental health problems in first-time mothers. Given the dearth of cost-effective preventive interventions in this field and the clinical, social and economic incentives to prevent postnatal mental health problems, further research is warranted to reduce the uncertainty surrounding the results.

Contributors JR, PL, TT, KW, HR and JF contributed to the design of the economic evaluation, interpretation of results and approval of the final paper; $\mathrm{JR}$ and PL undertook the data analyses and prepared the manuscript; JF and HR developed the WWWT intervention.

Funding This study was funded by competitively awarded grants from the National Health and Medical Research Council (APP1026550), the Australian Government Department of Social Services (formerly Families, Housing, Community Services and Indigenous Affairs) and the Victorian Department of Education and Early Childhood Development.

Competing interests None declared.

Ethics approval Southern Health (now Monash Health) Human Research Ethics Committee, also the Education and Policy Research Committee, Victorian Government Department of Education and Early Childhood Development (now Department of Education and Training) and the study was registered with the Monash University Human Research Ethics Committee.

Provenance and peer review Not commissioned; externally peer reviewed.

Data sharing statement No additional data are available.

Open Access This is an Open Access article distributed in accordance with the Creative Commons Attribution Non Commercial (CC BY-NC 4.0) license, which permits others to distribute, remix, adapt, build upon this work noncommercially, and license their derivative works on different terms, provided the original work is properly cited and the use is non-commercial. See: http:// creativecommons.org/licenses/by-nc/4.0/

\section{REFERENCES}

1. Gavin NI, Gaynes BN, Lohr KN, et al. Perinatal depression: a systematic review of prevalence and incidence. Obstet Gynecol 2005;106(Pt 1):1071-83.

2. Fisher J, Cabral de Mello M, Patel V, et al. Prevalence and determinants of common perinatal mental disorders in women in low- and lower-middle-income countries: a systematic review. Bull World Health Organ 2012;90:139G-49G.

3. Da Costa D, Dritsa M, Rippen N, et al. Health-related quality of life in postpartum depressed women. Arch Women Ment Health 2006:9:95-102

4. Reay R, Matthey S, Ellwood D, et al. Long-term outcomes of participants in a perinatal depression early detection program. $J$ Affect Disord 2011;129:94-103.

5. Dagher RK, McGovern PM, Dowd BE, et al. Postpartum depression and health services expenditures among employed women. J Occup Environ Med 2012;54:210-15.

6. Webster J, Pritchard MA, Linnane JW, et al. Postnatal depression: use of health services and satisfaction with health-care providers. J Qual Clin Pract 2001;21:144-8.

7. Petrou S, Cooper P, Murray L, et al. Economic costs of post-natal depression in a high-risk British cohort. $\mathrm{Br} J$ Psychiatry 2002;181:505-12.

8. MacArthur C, Winter HR, Bick DE, et al. Redesigning postnatal care: a randomised controlled trial of protocol-based midwifery-led care focused on individual women's physical and psychological health needs. Health Technol Assess 2003;7:1-98.

9. Petrou S, Cooper P, Murray L, et al. Cost-effectiveness of a preventive counseling and support package for postnatal depression. Int J Technol Assess 2006;22:443-53.

10. Dukhovny D, Dennis CL, Hodnett E, et al. Prospective economic evaluation of a peer support intervention for prevention of postpartum depression among high-risk women in Ontario, Canada. Am J Perinatol 2013;30:631-42.
11. Morrell CJ, Spiby $H$, Stewart $P$, et al. Costs and effectiveness of community postnatal support workers: randomised controlled trial. BMJ 2000;321:593-8.

12. Pilkington PD, Whelan TA, Milne LC. A review of partner-inclusive interventions for preventing postnatal depression and anxiety. Clin Psychol-UK 2015;19:63-75.

13. Fisher JR, Wynter KH, Rowe HJ. Innovative psycho-educational program to prevent common postpartum mental disorders in primiparous women: a before and after controlled study. BMC Public Health 2010;10:432.

14. Rowe $\mathrm{H}$, Wynter $\mathrm{K}$, Lorgelly $\mathrm{P}$, et al. A cluster randomised controlled trial of a brief couple-focused psychoeducational intervention to prevent common postnatal mental disorders among women: study protocol. BMJ Open 2014;4:e006436.

15. Department of Education and Early Childhood Development Victoria. Maternal and Child Health Service Program Standards, 2009.

16. Department of Education \& Training. Maternal and Child Health Annual Report Archive: State Government Victoria. 2014. http://www. education.vic.gov.au/childhood/providers/support/Pages/ mchannualreportarchive.aspx

17. Edgecombe G, White S, Marsh G, et al. First-time parent group resource and facilitation guide for maternal and child health nurses. Melbourne: Victorian Department of Human Services, 2001:1-120.

18. Department of Education and Early Childhood Development Victoria Municipal Association of Victoria. Memorandum of Understanding between Department of Education and Early Childhood Development AND Municipal Association of Victoria in relation to Maternal and Child Health Services July 2012-June 2015. VIC, Australia, 2012

19. Drummond MF, Sculpher MJ, Torrance GW, et al. Methods for the economic evaluation of health care programmes. 3rd edn. Oxford, UK: Oxford University Press, 2005.

20. Weinstein MC, Siegel JE, Gold MR, et al. Recommendations of the panel on cost-effectiveness in health and medicine. JAMA 1996;276:1253-8.

21. Unützer J, Katon WJ, Russo J, et al. Willingness to pay for depression treatment in primary care. Psychiatr Serv 2003;54:340-5

22. Schomerus $\mathrm{G}$, Angermeyer MC, Matschinger $\mathrm{H}$, et al. Public attitudes towards prevention of depression. J Affect Disord 2008;106:257-63.

23. Simoens S. Health economic assessment: cost-effectiveness thresholds and other decision criteria. Int J Environ Res Public Health 2010;7:1835-40.

24. Ryen L, Svensson M. The willingness to pay for a quality adjusted life year: a review of the empirical literature. Health Econ 2015;24:1289-301.

25. Shiroiwa T, Sung YK, Fukuda T, et al. International survey on willingness-to-pay (WTP) for one additional QALY gained: what is the threshold of cost effectiveness? Health Econ 2010;19:422-37.

26. NICE. Guide to the methods of technology appraisal 2013. UK NICE, 2013.

27. Claxton K, Martin S, Soares M, et al. Methods for the estimation of The National Institute for Health and Care Excellence cost-effectiveness threshold. Health Technol Assess 2015;19:1-503.

28. Ride J, Rowe H, Wynter K, et al. Protocol for economic evaluation alongside a cluster-randomised controlled trial of a psychoeducational intervention for the primary prevention of postnatal mental health problems in first-time mothers. BMJ Open 2014:4:e006226.

29. Fisher J, Rowe H, Wynter K, et al. Gender-informed, psychoeducational programme for couples to prevent postnatal common mental disorders among primiparous women: cluster randomised controlled trial. BMJ Open 2016;6:e009396.

30. Killip S, Mahfoud Z, Pearce K. What is an intracluster correlation coefficient? Crucial concepts for primary care researchers. Ann Fam Med 2004;2:204-8.

31. Australian Government Department of Health. MBS Online: Commonwealth of Australia. http://www.mbsonline.gov.au/internet/ mbsonline/publishing.nsf/Content/downloads

32. Independent Hospital Pricing Authority. National Efficient Price Determination 2013-14: Commonwealth of Australia. http://www.ihpa. gov.au/internet/ihpa/publishing.nsf/content/nep-determination-2013-14

33. American Psychiatric Association. Diagnostic and statistical manual-text revision (DSM-IV-TRim, 2000). American Psychiatric Association, 2000.

34. Viney R, Norman R, King MT, et al. Time trade-off derived EQ-5D weights for Australia. Value Health 2011;14:928-36.

35. Brooks R. EuroQol: the current state of play. Health Policy 1996;37:53-72 
36. Wisløff T, Hagen G, Hamidi V, et al. Estimating QALY gains in applied studies: a review of cost-utility analyses published in 2010 . Pharmacoeconomics 2014;32:367-75.

37. Gutke A, Lundberg M, Östgaard HC, et al. Impact of postpartum lumbopelvic pain on disability, pain intensity, health-related quality of life, activity level, kinesiophobia, and depressive symptoms. Eur Spine J 2011;20:440-8.

38. Shorten A, Shorten B. The importance of mode of birth after previous cesarean: success, satisfaction, and postnatal health J Midwifery Womens Health 2012;57:126-32.

39. Jansen AJ, Essink-Bot ML, Duvekot JJ, et al. Psychometric evaluation of health-related quality of life measures in women after different types of delivery. J Psychosom Res 2007;63:275-81.

40. Turkstra E, Gamble J, Creedy DK, et al. PRIME: impact of previous mental health problems on health-related quality of life in women with childbirth trauma. Arch Womens Ment Health 2013;16:561-4.

41. Petrou S, Morrell J, Spiby H. Assessing the empirical validity of alternative multi-attribute utility measures in the maternity context. Health Qual Life Outcomes 2009;7:40.

42. Australian Bureau of Statistics. Socio-Economic Index for Areas. 2013. http://www.abs.gov.au/websitedbs/censushome.nsf/home/seifa

43. Glick HA, Doshi JA, Sonnad SS, et al. Economic evaluation in clinical trials. 2nd edn. Oxford, UK: Oxford University Press, 2015.

44. Briggs $\mathrm{AH}$. Statistical approaches to handling uncertainty in health economic evaluation. Eur J Gastroenterol Hepatol 2004;16:551-61.

45. Black WC. The CE plane a graphic representation of cost-effectiveness. Med Decis Making 1990;10:212-14.

46. Fenwick E, Byford S. A guide to cost-effectiveness acceptability curves. Br J Psychiatry 2005;187:106-8.

47. Kirkwood B, Sterne J. Essential medical statistics: John Wiley \& Sons, Ltd, 2003.

48. Claxton K. The irrelevance of inference: a decision-making approach to the stochastic evaluation of health care technologies. J Health Econ 1999;18:341-64.
49. Morrell CJ, Warner R, Slade P, et al. Psychological interventions for postnatal depression: cluster randomised trial and economic evaluation. The PoNDER trial. Health Technol Assess 2009;13:iii-iv, xi-xiii, 1-153.

50. Fisher JRW, Feekery CJ, Rowe-Murray HJ. Nature, severity and correlates of psychological distress in women admitted to a private mother-baby unit. J Paediatr Child Health 2002;38:140-5.

51. White IR, Carlin JB. Bias and efficiency of multiple imputation compared with complete-case analysis for missing covariate values. Stat Med 2010;29:2920-31.

52. Brazier J. Is the EQ-5D fit for purpose in mental health? Br J Psychiatry 2010;197:348-9.

53. Mihalopoulos $\mathrm{C}$, Chen $\mathrm{G}$, lezzi $\mathrm{A}$, et al. Assessing outcomes for cost-utility analysis in depression: comparison of five multi-attribute utility instruments with two depression-specific outcome measures. Br J Psychiatry 2014;205:390-7.

54. Willan AR, O'Brien BJ. Sample size and power issues in estimating incremental cost-effectiveness ratios from clinical trials data. Health Econ 1999;8:203-11.

55. Manju MA, Candel MJ, Berger MP. Sample size calculation in cost-effectiveness cluster randomized trials: optimal and maximin approaches. Stat Med 2014;33:2538-53.

56. Roberts SL, Bushnell JA, Collings SC, et al. Psychological health of men with partners who have post-partum depression. Aust N Z $J$ Psychiatry 2006;40:704-11.

57. Australian Bureau of Statistics. Pregnancy and work transitions. 2013 (20 November 2015). http://www.abs.gov.au/ausstats/abs@ . nsf/Lookup/4102.0Main+Features10Nov+2013

58. Spitzer RL, Kroenke K, Williams JB. Validation and utility of a self-report version of PRIME-MD: the PHQ primary care study. Primary Care Evaluation of Mental Disorders. Patient Health Questionnaire. JAMA 1999;282:1737-44.

59. Spitzer RL, Kroenke K, Williams JB, et al. A brief measure for assessing generalized anxiety disorder: the GAD-7. Arch Intern Med 2006;166:1092-7. 